\title{
Sonosensitive dioleoylphosphatidylethanolamine-containing liposomes with prolonged blood circulation time of doxorubicin
}

Tove J. Evjen ${ }^{\mathrm{a}, \mathrm{b}}$, Eirik Hagtvet ${ }^{\mathrm{c}}$, Esben A. Nilssen ${ }^{\mathrm{a}^{*}}$, Martin Brandl ${ }^{\mathrm{b} 2}$, Sigrid L. Fossheim ${ }^{\mathrm{a} 3}$.

\begin{abstract}
${ }^{a}$ Epitarget AS, Oslo, Norway. ${ }^{b}$ University of Tromsø, Department of Pharmacy, Tromsø, Norway. ${ }^{\mathrm{c}}$ Department of Radiation Biology, Institute for Cancer Research, Oslo University Hospital and University of Oslo, Oslo, Norway. ${ }^{2}$ Current address: University of Southern Denmark, Department of Chemistry and Physics, Odense, Denmark. ${ }^{3}$ Current address: Clavis Pharma ASA, Oslo, Norway.

*Corresponding author. Epitarget AS, PO Box 7169 Majorstuen, 0307 Oslo, Norway. E-mail: esben.nilssen@epitarget.com
\end{abstract}

\begin{abstract}
Ultrasound sensitive (sonosensitive liposomes) are drug delivery systems designed for releasing their drug load upon exposure to ultrasound (US). Inclusion of dioleoylphosphatidylethanolamine (DOPE) in liposome membranes was previously shown to induce sonosensitivity. For efficient US mediated drug delivery to solid tumours, a long blood circulation time of the liposomal drug providing high tumour uptake is required. In this study, blood pharmacokinetics of DOPE-based liposomal doxorubicin (DXR) were evaluated in mice. A markedly faster blood clearance of DXR was observed for DOPE-rich liposomes compared to Caelyx ${ }^{\circledR}$ (standard liposomal DXR). Subsequently, liposome membrane composition was altered to improve drug retention in the bloodstream, while maintaining sonosensitivity. Formulations with reduced blood clearance of DXR were obtained by reducing the content of DOPE from 62 to 32 or $25 \mathrm{~mol} \%$. These formulations showed long blood circulation time, as approximately $20 \%$ of the administered DXR dose was present in the bloodstream $24 \mathrm{~h}$ after intravenous injection. The reduction in liposomal DOPE content did not significantly reduce US mediated DXR release in vitro, indicating that DOPE is a potent modulator to sonosensitivity. The novel liposome formulations, containing moderate amounts of DOPE, displayed similar blood pharmacokinetic profiles as standard liposomal DXR, but a markedly improved sonosensitivity.
\end{abstract}

\section{Keywords}

Liposomes; Doxorubicin; Pharmacokinetics; Ultrasound; Sonosensitive; Drug delivery. 


\section{INTRODUCTION}

Liposomes that exhibit controlled release properties have potential for delivery of chemotherapeutic drugs to solid tumours. Ideally, liposomes should accumulate intact at the tumour site, and subsequently release their drug load. The extent of liposome accumulation in the tumour is largely determined by the blood circulation time of the liposomal drug, where liposomes that retain their drug over several hours to days will provide greater tumour delivery and less non-specific toxicity. For reviews see Drummond et al., 1999; Gabizon et al., 2003. After liposome accumulation within the tumour tissue, the encapsulated drug must be released before it can be taken up by the neoplastic cells (Horowitz et al., 1992). In the case of long circulating PEGylated doxorubicin (DXR)-liposomes, the drug has shown to leak slowly from the liposome (Horowitz et al., 1992; Colbern et al., 2000). Thus, triggered destabilization of the liposome carrier within the tumour area might lead to a substantial increase in drug availability and thus improved therapeutic efficacy.

Recently, there has been increasing interest in using ultrasound (US) as a means to physically and non-invasively trigger drug release from liposomes upon accumulation in tumour tissue (de Smet et al., 2010; Schroeder et al., 2009; Myhr and Moan, 2006; Pong et al., 2006). Local application of US to tumours produces several effects, such as enhancing extravasation of liposomes to tumours, inducing drug leakage from the liposome carrier and enhancing cell membrane permeability with a resulting increased intracellular drug uptake (For recent reviews see Schroeder et al., 2009; Pitt et al., 2004; Frenkel, 2008). In order to maximize the therapeutic efficacy, liposomes should display properties that both impart high US sensitivity (sonosensitivity) and favour drug retention in the blood stream, to enable sufficient tumour accumulation. Several studies have shown that gas-containing particles are highly sonosensitive (Huang et al., 2008; Unger et al., 1998; Huang and MacDonald., 2004). Unfortunately, gas-filled liposomes are micron-sized and thus too large to allow for effective extravasation into tumour tissue (Ferrara, 2008; Maeda et al., 2000). Another limitation of such particles is the rapid dissolution of the entrapped gas in blood circulation with consequent loss of sonosensitivity (Unger et al., 1998; Ferrara, 2008).

An approach to formulate gas-free liposomes combining high sonosensitivity with long blood circulations times might be to modulate the liposome membrane composition. Previously, we demonstrated that PEGylated liposomes comprising phosphatidylethanolamine showed a several fold increase in drug release compared to Caelyx ${ }^{\circledR}$ (standard liposomal DXR) during in vitro exposure to US (Evjen et al., 2010; Evjen et al., 2011a; Evjen et al., 2011b). An US enhanced therapeutic activity of DSPE-based liposomal DXR was demonstrated in tumoured mice (Hagtvet et al., 2010). Recently, we 
found that liposomes based on DOPE as the main phospholipid constituent displayed markedly improved sonosensitivity than DSPE-based liposomes (Evjen et al., 2011a). These DOPE-based liposomes, however, were not evaluated and optimized as drug carriers for in vivo applications.

In the present study, blood circulation time of various DXR-containing liposome formulations combining DOPE with distearoylphosphatidylcholine (DSPC), PEGylated distearoylphosphatidylethanolamine (DSPE-PEG 2000) and cholesterol, were investigated in non-tumoured mice. Novel formulations combining high sonosensitivity and long blood residence time of DXR were obtained.

\section{MATERIALS AND METHODS}

\subsection{Materials}

Cholesterol, HEPES (4-(2-hydroxyethyl)-1-piperazineethanesulfonic acid) ammonium sulfate, TritonX-100® solution and sucrose were obtained from Sigma Aldrich, Oslo, Norway. Serum of fetal bovine origin (Autonorm) was obtained from Sero (Billingstad, Norway). DSPE-PEG 2000) DSPC, DOPE, were purchased from Genzyme Pharmaceuticals, Liestal, Switzerland. Doxorubicin hydrochloride (DXR) was purchased from Nycomed, Asker, Norway. DXR-containing liposomes, Caelyx ${ }^{\circledR}$ (herein defined as standard liposomal DXR), comprising hydrogenated soy phosphatidylcholine (HSPC), DSPE-PEG 2000 and cholesterol (57:5:38 mol \%) was supplied from the pharmacy at the Norwegian Radium Hospital, Oslo, Norway (European distributor Schering-Plough).

For anaesthesia of mice a mixture of $2.4 \mathrm{mg} / \mathrm{ml}$ tiletamine and $2.4 \mathrm{mg} / \mathrm{ml}$ zolazepam (Zoletil $^{\circledR}$ vet, Virbac Laboratories, Carros, France), $3.8 \mathrm{mg} / \mathrm{ml}$ xylazine (Narcoxyl ${ }^{\circledR}$ vet, Roche, Basel, Switzerland) and $0.1 \mathrm{mg} / \mathrm{ml}$ butorphanol (Torbugesic $^{\circledR}$, Fort Dodge Laboratories, Fort Dodge, IA) was prepared and used.

\subsection{Liposome preparation}

Liposomes with different mol \% of the lipids DOPE, DSPC, DSPE-PEG and cholesterol were prepared by the thin film hydration and sequential extrusion method similarly as previously described (Evjen et al., 2011a). (See Table 1, Section 3.2 for liposome membrane compositions). Briefly, dry lipid films were hydrated with $300 \mathrm{mM}$ ammonium sulfate solution and exposed to three freeze/thaw cycles in a dry-ice/aceton/methanol mixture and water, respectively. The liposomes were reduced in size by stepwise extrusion (Lipex extruder, Biomembrane Inc., Vancouver B.C., Canada) through Nucleopore polycarbonate 
filters with pore sizes of $800,400,200,100$ and $80 \mathrm{~nm}$ (Nuclepore, West Chester, PA, USA). The mean target size of the liposomes was $85 \pm 5 \mathrm{~nm}$.

DXR was remote loaded into liposomes using an ammonium sulfate transmembrane gradient (Haran et al., 1993), as previously described by Evjen et al., 2011a. The resulting nominal lipid and DXR concentrations were $16 \mathrm{mg} / \mathrm{ml}$ and $1 \mathrm{mg} / \mathrm{ml}$, respectively.

\subsection{Liposome characterization}

The mean intensity-weighted hydrodynamic liposome diameter was determined by Photon Correlation Spectroscopy (Nanosizer, Malvern Instruments, Malvern, UK). The liposome dispersions were diluted prior to measurements in a ratio of 1:200 (v/v) with 0.22 $\mu \mathrm{m}$ - filtered sucrose/HEPES solution. The measurements were performed at $23{ }^{\circ} \mathrm{C}$ and at a scattering angle of $90^{\circ}$. The polydispersity index (P.I) was given as an indicator for the width of the particle size distribution. Triplicate measurements of each liposome batch were performed.

Determination of liposomal entrapment efficiency of DXR was estimated by fluorescence measurements as previously described (Evjen et al., 2011). All liposome formulations displayed entrapment efficiencies of DXR of more than 95\% (drug:lipid ratio $1: 16 \mathrm{wt} / \mathrm{wt})$.

\subsection{Ultrasound mediated drug release}

US release measurements were conducted using a $40 \mathrm{kHz}$ US transducer (VC 750, Sonic and Materials, Inc, Newtown, CT, USA) with a $19 \mathrm{~mm}$ diameter nonfocused probe mounted in a custom-built sample chamber as previously described (Evjen et al., 2010). In brief, the liposome dispersions were diluted in a 1:500 (v/v) ratio with sucrose/HEPES solution containing $20 \%$ serum, just prior to the US experiments. The diluted liposome dispersions were exposed to $40 \mathrm{kHz}$ US at a nominal intensity of $12 \mathrm{~W} / \mathrm{cm}^{2}$ in a continuous mode $(100 \%$ duty cycle) up to $6 \mathrm{~min}$. Acoustic pressure measurements conducted with a Bruel and Kjaer hydrophone (Type 8103, Denmark) in the sample chamber gave $240 \mathrm{kPa}$ (pk-pk). The temperature in the liposome samples never exceeded $30{ }^{\circ} \mathrm{C}$ during the US experiments, excluding the possibility of direct thermal effect of US on liposomal drug release.

DXR release could be monitored due to the relief of DXR-mediated fluorescence self-quenching in the external liposomal phase, and concomitant increase in fluorescence intensity. An optical fiber probe (Ocean optics, type QP600-2-VIS/BX, Florida, USA) was 
used to measure in line the increase in fluorescence intensity as a result of DXR release, using the following equation:

Equation 1: $\quad \%$ Drug release $=\left(\mathrm{F}_{\mathrm{t}}-\mathrm{F}_{0}\right) /\left(\mathrm{F}_{\max }-\mathrm{F}_{0}\right) * 100$

Where $\mathrm{F}_{0}$ and $\mathrm{F}_{\mathrm{t}}$ are the fluorescence intensities of a given liposome sample prior to and after a given duration $(t)$ of US respectively, and $F_{\max }$ is the fluorescence intensity after liposome solubilisation with surfactant (Triton-X 100). The diluted liposome samples were solubilised with Triton-X 100 at a ratio of 100:1 (v/v). Fluorescence intensity measurements were performed using a fluorescence spectrometer from Ocean Optics (model QE65000. Duiven, Netherlands). The excitation and emission wavelength for DXR were 488 and $595 \mathrm{~nm}$, respectively. Triplicate samples were measured.

\subsection{Serum stability assay}

Liposome stability in vitro was studied using a well-established serum-induced leakage assay as previously described by Evjen et al., 2010. Liposome dispersions, diluted 1:125 (v/v) with sucrose/HEPES solution containing $20 \%$ serum were incubated up to $24 \mathrm{~h}$ at $37{ }^{\circ} \mathrm{C}$. Time-dependent leakage of DXR was quantified by fluorescence measurements, by further diluting the samples 1:4 (v/v) with sucrose/HEPES solution, according to Equation 1. Triplicate samples were measured.

\subsection{Pharmacokinetic and biodistribution studies in non-tumoured mice}

Male atymic nude Balb/c mice were provided by the Department of Comparative Medicine (animal facility), the Norwegian Radium Hospital. The mice were housed in transparent boxes with bedding material, fed ad libitum and kept under specific pathogen-free conditions. The temperature and relative humidity were kept constant at $20-21^{\circ} \mathrm{C}$ and $60 \%$, respectively. At the end of the experiments all animals were euthanized by cervical dislocation. All procedures were performed according to protocols approved by the National Animal Research Authority and carried out in compliance with the European Convention for the Protection of Vertebrates Used for Scientific Purposes.

The administered DXR dose for animals receiving the various DOPE-based liposomes was $7 \mathrm{mg} / \mathrm{kg}$ body weight. The DXR dose for animals receiving standard liposomal DXR was $14 \mathrm{mg} / \mathrm{kg}$. The doubled DXR dose administered was due to the doubled drug-to lipid-ratio of the standard liposomes (1:8 drug/lipid) versus DOPE-based liposomes (1:16 drug/lipid). Thus, the dosing was based on an identical lipid dose for all investigated liposome 
formulations, as the lipid dose (i.e. liposome dose) is generally known as a key factor influencing blood pharmacokinetics (Drummond et al., 1999).

The liposomes were injected intravenously (i.v.) into the tail vein under anaesthesia induced by subcutaneous administration of $0.1 \mathrm{ml}$ anaesthetic agent. Animals were sacrificed in groups of three at different time points post injection $(0.5,1,3,8$ 12, 24, $48 \mathrm{~h})$. Blood samples were obtained by cardiac puncture using heparinized syringes and stored in heparinized tubes. Liver, spleen and kidneys were excised and their weight registered. All blood and tissue samples were kept on ice bath until storage at $-80{ }^{\circ} \mathrm{C}$.

Quantification of DXR was performed similarly as described by Gabizon et al., 1989. In brief, $0.1 \mathrm{ml}$ of whole blood samples (lysed due to freezing) was mixed with $1.9 \mathrm{ml}$ acidified ethanol $(0.3 \mathrm{M} \mathrm{HCl}$ in 50\% ethanol) giving a final 1:20 (v/v) dilution. Duplicate samples were prepared for further processing. Tissue samples were added $50 \%$ acidified ethanol in a final 1:10 (v/v) dilution and homogenized using a Homogenizer PRO 200® (PRO Scientific, Monroe, CT, USA). The digested blood and tissue samples were incubated for $24 \mathrm{~h}$ at $4{ }^{\circ} \mathrm{C}$ in the dark. Following incubation the precipitate was removed by centrifugation $\left(20000 \mathrm{~g}, 20 \mathrm{~min}, 4{ }^{\circ} \mathrm{C}\right)$ and the supernatant (containing extracted DXR) stored at $-20^{\circ} \mathrm{C}$ until fluorescence measurements were performed. The extracted DXR was quantified by fluorescence measurements as previously described using multipoint standard curves. The latter were generated by adding known amounts of standard liposomal DXR to blood and tissue (liver, spleen and kidney) homogenate and incubated and centrifuged as described above. All calibration curves had regression coefficients $\left(\mathrm{R}^{2}\right)>0,99$.

\subsection{Statistics}

All data are reported as mean values with standard deviation. Statistical comparison of the DXR concentration in blood and organs for the different liposome formulations was performed at each time point post injection using a student $\mathrm{t}$-test with $\mathrm{p}<0.05$.

\section{RESULTS}

\subsection{Blood pharmacokinetics of prototype DOPE-based liposomes}

DOPE-based liposomes with membrane composition DOPE:DSPC:DSPE-PEG 2000:CHOL 62:10:8:20 mol\% were previously demonstrated to release their DXR load almost completely during 4 minutes of US exposure in vitro (Evjen et al., 2011). Furthermore these liposomes showed negligible leakage of DXR during $24 \mathrm{~h}$ in vitro incubation in sucrose/HEPES solution containing $20 \%$ serum at $37^{\circ} \mathrm{C}$ (Evjen et al., 2011). 
Hence, this DOPE-based formulation was first selected for assessment of blood pharmacokinetics in mice. Blood levels of DXR provide a good indication of liposomal drug retention, since DXR released in the bloodstream is rapidly distributed to other tissues and organs (Gabizon et al., 1989).

Fig 1 shows the blood clearance profiles of DXR for DOPE-based liposomes in comparison to standard liposomal DXR. Both formulations showed exponential declines in blood concentrations, starting with a rapid decline in DXR blood concentrations followed by a slower elimination phase. In the first distribution phase, DXR concentrations were comparable between the two formulations. In the slower elimination phase, starting approximately at $3 \mathrm{~h}$ post injection, a significantly lower blood concentration was observed for the DOPE-based liposomes as compared to standard liposomal DXR. At $24 \mathrm{~h}$ post injection only $5 \%$ of the injected DXR dose remained in blood for the DOPE-based liposomes versus $20 \%$ for standard liposomal DXR (Fig 1).

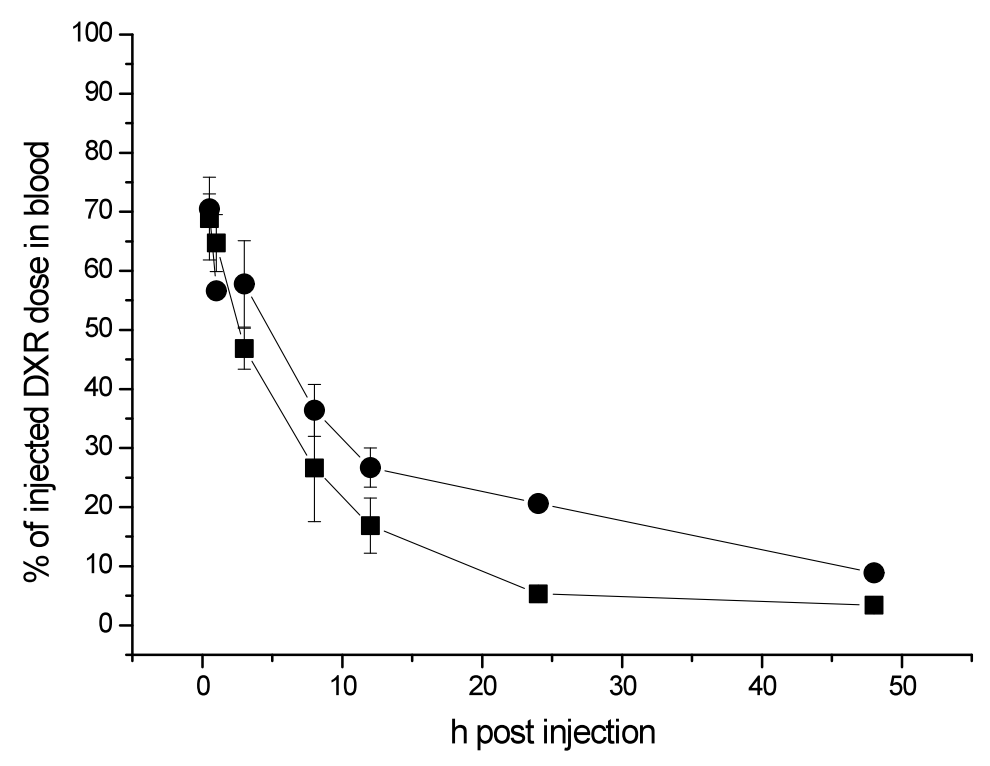

Fig 1. Blood clearance kinetics of i.v. injected liposomal DXR in mice, - DOPE-based liposomes (DOPE:DSPC:DSPE-PEG 2000:Chol; 62:10:8:20 mol\%) in comparison to standard liposomal DXR (HSPC:DSPE-PEG 2000:Chol; 57:5:38 mol\%). Bars represent the $\mathrm{SD}$ of the mean $(\mathrm{n}=3)$. 


\subsection{Effect of lipid composition on DXR blood pharmacokinetics of DOPE-based liposomes}

Further, both blood clearance kinetics and in vitro sonosensitivity for a range of DOPE-based formulations with different lipid composition were investigated. Unsaturated phospholipids, like DOPE, fluidize the liposome membrane thereby increasing the membrane permeability at physiological temperature due to their low gel-to-liquid-crystalline phase transition temperature $\left(T_{c}\right)$ (Demel et al., 1976). Previous studies have shown that addition of cholesterol to liposomes rigidifies the bilayer and reduces leakage of entrapped material at temperatures above the $T_{\mathrm{c}}$ of the membrane (Gregoriadis, 1988; Damen et al., 1981; Ishida et al., 2006).

In an attempt to prolong blood circulation time of DOPE-containing liposomal DXR, membrane composition was altered by both increasing the level of cholesterol and reducing the level of DOPE. An overview of the different formulations produced is presented in Table 1.

Note that all formulations were comparable in terms of mean size and drug-to-lipid ratio, and differed only in membrane composition. The comparable liposome size was essential to better assess the influence of the membrane composition per se on blood circulation time and biodistribution. In vitro leakage studies showed no detectable leakage of DXR from the liposomes during the course of $24 \mathrm{~h}$ incubation in $20 \%$ serum at $37^{\circ} \mathrm{C}$.

Table 1. Overview of DXR-containing DOPE-liposomes.

\begin{tabular}{ccccc}
\hline no & $\begin{array}{c}\text { DOPE:DSPC:DSPE-PEG:Chol } \\
(\mathbf{m o l} \%)\end{array}$ & $\begin{array}{c}\text { Mean size, } \\
\text { nm (P.I) }\end{array}$ & $\begin{array}{c}\text { \% DXR in } \\
\text { plasma } \\
\text { 24 post inj. }\end{array}$ & $\begin{array}{c}\text { \% DXR } \\
\text { released } \\
\text { 6 min post US }\end{array}$ \\
\hline 1 & $62: 10: 8.20$ & $86(0.14)$ & $5 \pm 1$ & $91 \pm 2$ \\
2 & $52: 5: 8: 35$ & $86(0.12)$ & $3 \pm 1$ & $79 \pm 1$ \\
3 & $52: 20: 8: 20$ & $80(0.10)$ & $4 \pm 1$ & $78 \pm 6$ \\
4 & $52: 0: 8: 40$ & $86(0.10)$ & $2 \pm 0$ & $74 \pm 1$ \\
5 & $32: 20: 8.40$ & $86(0.10)$ & $20 \pm 3$ & $87 \pm 2$ \\
6 & $25: 27: 8: 40$ & $87(0.10)$ & $17 \pm 1$ & $71 \pm 4$ \\
7 & $12: 40: 8: 40$ & $83(0.10)$ & $\mathrm{NA}$ & $11 \pm 1$ \\
8 & Standard liposomal DXR & $76(0.11)$ & $21 \pm 1$ & $3 \pm 1$ \\
\hline
\end{tabular}

At a constant DOPE level of $52 \mathrm{~mol} \%$ no change in blood clearance were observed for liposomes containing different cholesterol levels up to $40 \mathrm{~mol} \%$ (formulation no 1-4) (Fig 2). In contrast, liposomal DXR containing an intermediate level of DOPE (32 and 25 mol\%, respectively), and consequently an increased level of DSPC (20 and 27 mol\%, respectively), showed a significant prolonged circulation time (Fig 3). The blood clearance profiles of DXR 
for these intermediate DOPE formulations were approximately similar to standard liposomal DXR, where about $20 \%$ of the injected DXR dose remained in blood circulation $24 \mathrm{~h}$ after injection (Fig 3).

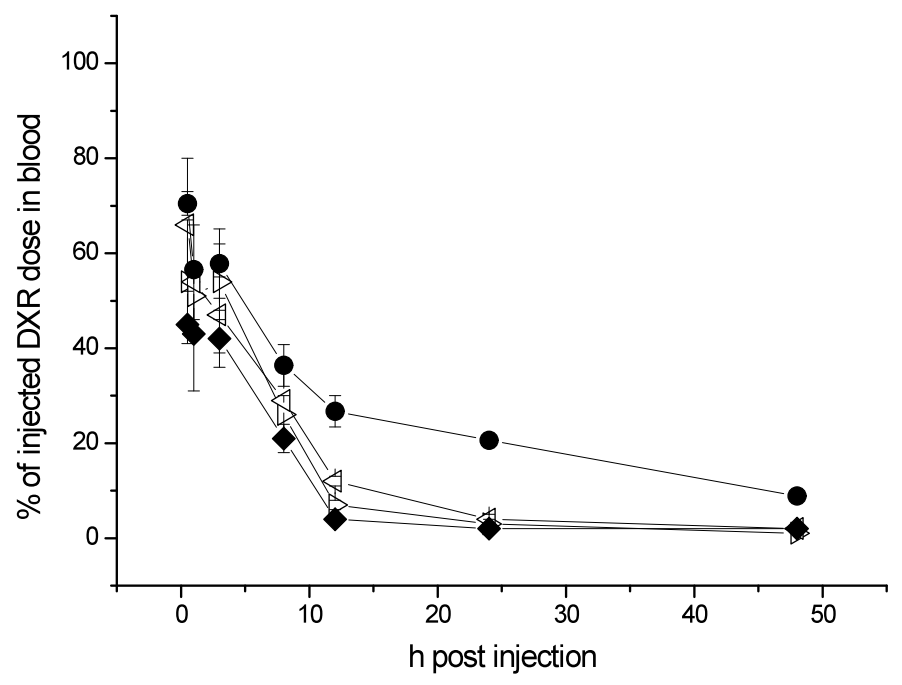

Fig 2. Blood clearance kinetics of i.v. injected liposomal DXR in mice of liposomes containing the following cholesterol levels; $\triangleleft 20 \mathrm{~mol} \%, \triangleright 35 \mathrm{~mol} \%$, $40 \mathrm{~mol} \%$. (DOPE and DSPE-PEG 2000 levels: 52 and $8 \mathrm{~mol} \%$, respectively. DSPC level co varies with cholesterol level). • standard liposomal DXR (HSPC:DSPE-PEG 2000:Chol; 57:5:38 mol\%) is included for comparison. Bars represent the SD of the mean $(n=3)$. 


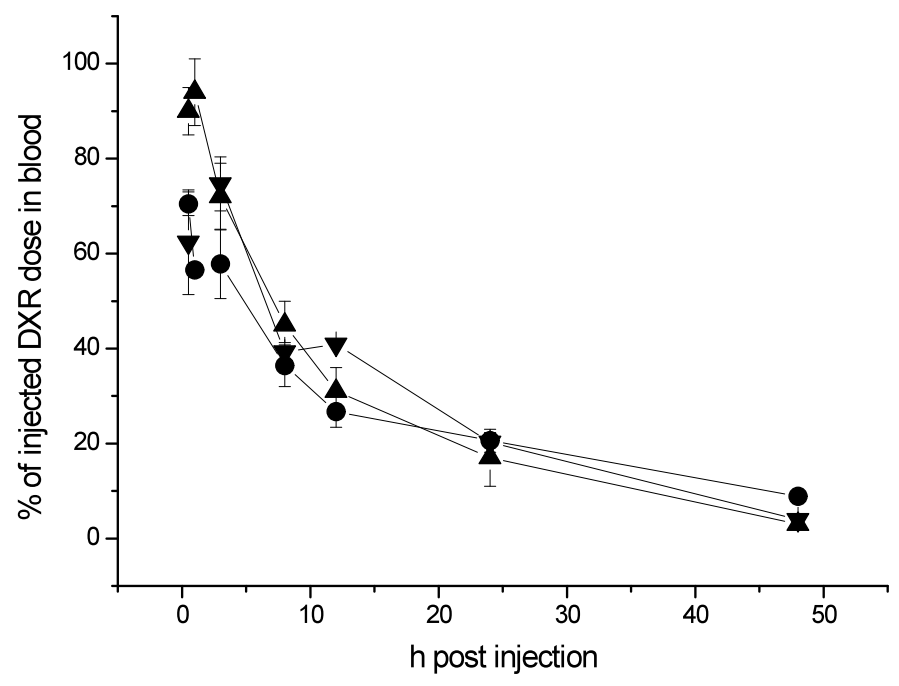

Fig 3. Blood clearance kinetics of i.v. injected liposomal DXR in mice of liposomes containing the following DOPE levels; $\boldsymbol{\Delta} 25 \mathrm{~mol} \%$ and $\nabla 32 \mathrm{~mol} \%$. (Cholesterol and DSPEPEG 2000 levels: 40 and 8 mol\%, respectively. DSPC level co varies with DOPE level). standard liposomal DXR (HSPC:DSPE-PEG 2000:Chol; 57:5:38 mol\%) is included for comparison. Bars represent the SD of the mean $(n=3)$.

\subsection{Effect of lipid composition on biodistribution of DOPE-containing liposomal DXR}

If the relatively faster blood clearance of DXR for DOPE-rich liposomes was a consequence of carrier recognition by the mononuclear phagocyte system (MPS), then a corresponding increased accumulation in liver and spleen would be expected._Hence, DXR levels in liver, spleen and kidneys were investigated for two formulations comprising 32 and $52 \mathrm{~mol} \%$ DOPE, respectively (no 4 and 5). These two formulations, herein termed intermediatelevel and highlevel DOPE-containing liposomes, showed a relatively slow and fast blood clearance of DXR, respectively (Fig 2 and 3).

No significant differences in DXR concentration in spleen could be observed for the intermediate and highlevel DOPE formulations (Fig 4B). The data revealed a slightly lower DXR concentration in liver for the highlevel DOPE-containing liposomes (Fig 4A). However, the difference was only significant at $12 \mathrm{~h}$ and $48 \mathrm{~h}$ post injection, $\mathrm{p}<0.05$ (Fig 4A). Thus, the faster blood clearance profile of DXR for this formulation most likely reflected leakage of DXR from the liposome carrier, and not an increased uptake by the MPS. 
Moreover, for the highlevel DOPE-containing liposomes, DXR levels in kidneys reached a maximum $12 \mathrm{~h}$ after injection. The intermediatelevel DOPE-containing liposomes showed sustained levels of DXR throughout the course of the experiment (Fig 4C). This pattern may reflect slow release of DXR from the intermediatelevel DOPE-containing liposomes in blood circulation, as indicated in Figs 2 and 3, resulting in higher DXR concentrations in the kidneys at the later time-points (Fig 4C).

$\mathbf{A}$

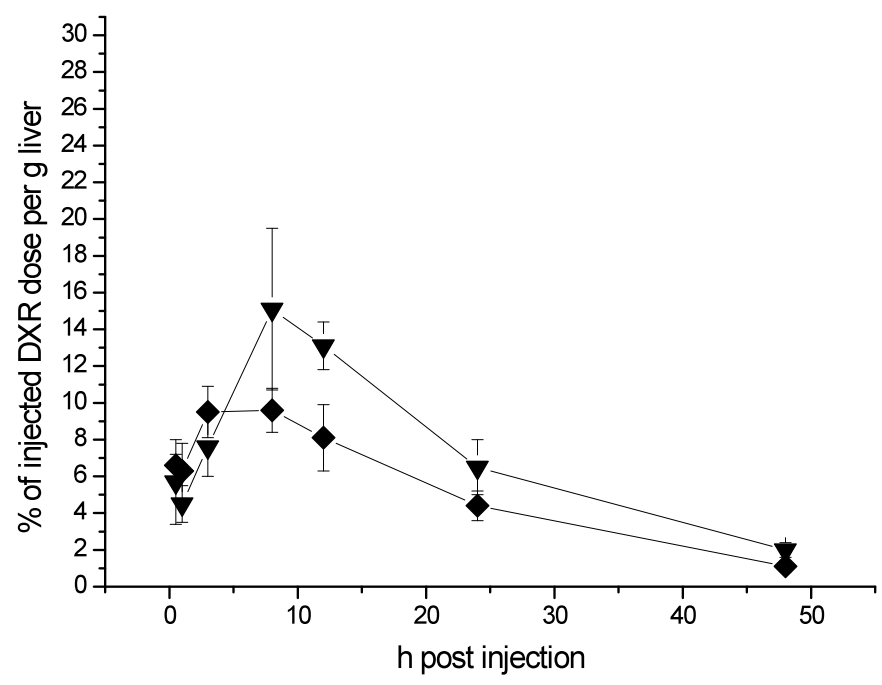

B

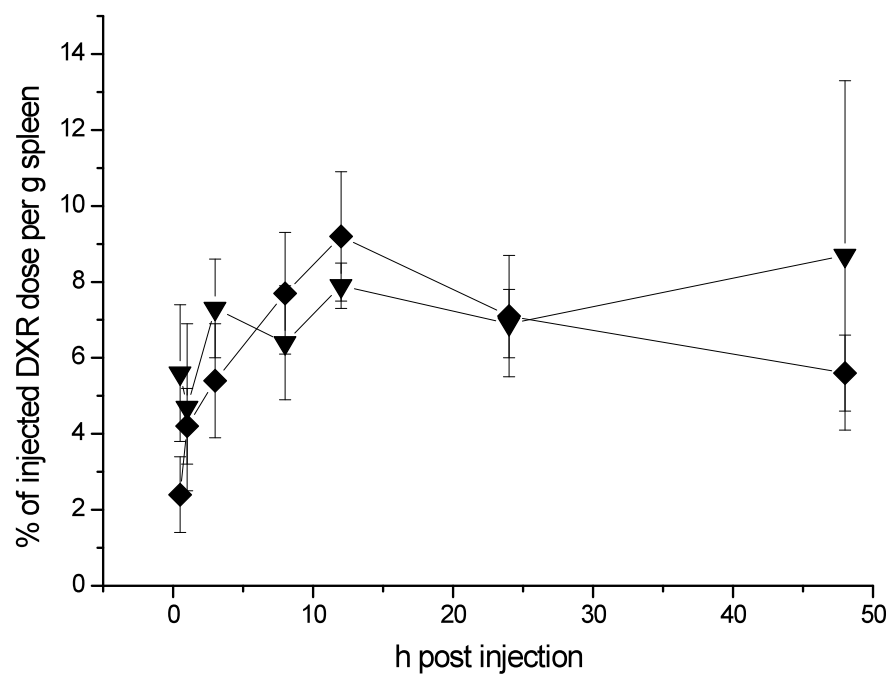




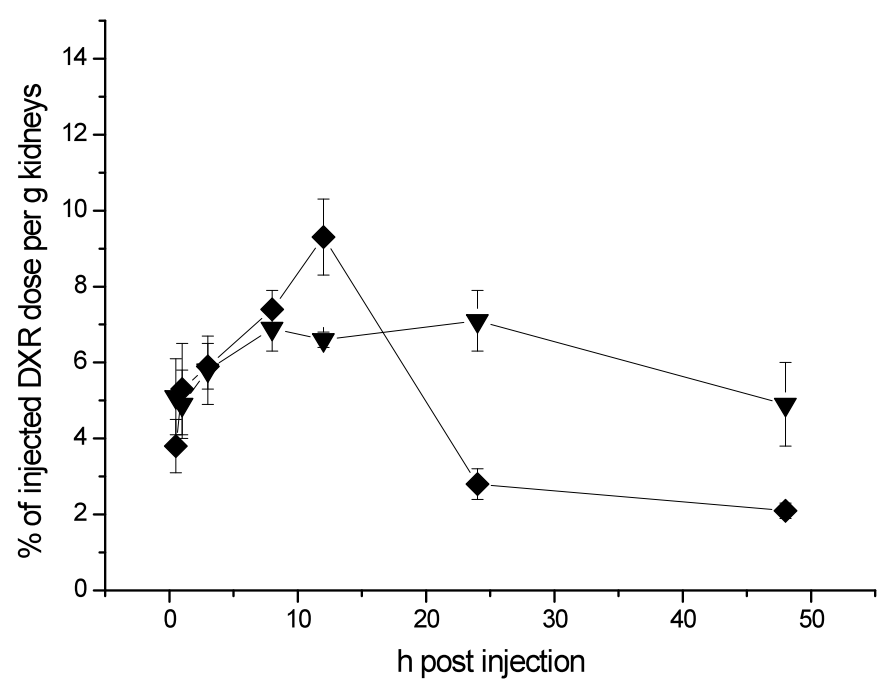

Fig 4. \% DXR of injected dose in (A) liver, (B) spleen and (C) kidneys after i.v. injection of DOPE:DSPC:DSPE-PEG 2000:Cholesterol liposomes in mice: 52:0:8:40 mol\%, $\boldsymbol{\nabla} 32: 20: 8: 40 \mathrm{~mol} \%$. Bars represent SD of the mean $(\mathrm{n}=3)$.

\subsection{Effect of DOPE level on sonosensitivity}

Variations in lipid membrane composition are shown to influence sonosensitivity of liposomes (Pong et al., 2006; Schroeder et al., 2009; Evjen et al., 2010a; Evjen et al., 2010b; Lin et al., 2001). We recently demonstrated that incorporation of DOPE into liposome bilayers drastically enhanced sonosensitivity compared to DSPE-based liposomes and standard liposomal DXR, respectively (Evjen et al., 2011a). The minimum amount of DOPE required for maintaining an acceptable sonosensitivity in vitro, however, was not explored.

Fig 5 compares the US mediated DXR release profiles of liposomes containing different amounts of DOPE, during exposure to $40 \mathrm{kHz}$ US in sucrose/HEPES solution containing $20 \%$ serum. High sonosensitivity was observed for formulations comprising between 25 and $52 \mathrm{~mol} \%$ DOPE (Fig 5). These formulations showed up to a 7-fold increase in DXR release extent when compared to standard liposomal DXR after 6 min US exposure. Poor sonosensitivity was observed for liposomes composed of $12 \mathrm{~mol} \%$ DOPE, as evidenced by a significant reduction in DXR release (Fig 5). 


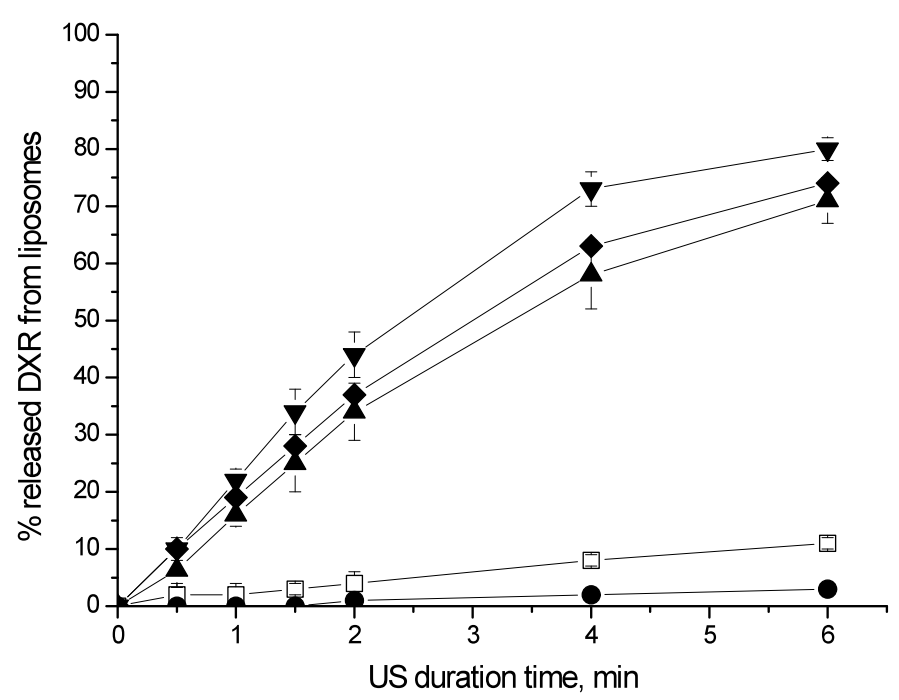

Fig 5. Effect of DOPE level on US mediated DXR release from liposomes in HEPES/sucrose solution containing 20\% serum (40 kHz US). DOPE-levels: $\boldsymbol{\nabla} 32 \mathrm{~mol} \%$, $52 \mathrm{~mol} \%$, \ 25 mol\%, $\square 12 \mathrm{~mol} \%$. (Cholesterol and DSPE-PEG 2000 levels: 40 and $8 \mathrm{~mol} \%$, respectively. DSPC level co varies). • standard liposomal DXR (HSPC:DSPE-PEG 2000:Chol; 57:5:38 mol\%) is included for comparison. Bars represent the SD of the mean of triplicate measurements.

\section{DISCUSSION}

Application of sonosensitive liposomes for US mediated drug delivery requires that the liposomes retain their drug contents in blood circulation until they reach the tumour tissue. The results of the current study showed that DOPE-containing liposomes combining long blood circulation time of DXR with sufficient sonosensitivity might be obtained by altering liposome membrane composition.

The results demonstrated that lipid composition significantly influenced the blood clearance kinetics of DOPE-based liposomal DXR. The in vivo blood clearance of liposomal drug is mainly determined by two factors: (1) uptake of intact liposomes by the MPS, (2) leakage of the entrapped drug from the liposomes in blood circulation. In general, small PEGylated liposomes below $100 \mathrm{~nm}$ in diameter, as described in the current study, are to a certain extent able to escape the MPS, which in turn provides prolonged blood circulation time of the liposome carrier and consequently increased accumulation within tumour tissue. For reviews see Drummond et al., 1999; Gabizon et al., 2003. For successful drug delivery, it is required that the liposomes are able to retain the entrapped drug in blood circulation en route to the tumour tissue. 
The liposomes comprising high levels of DOPE (62 and 52 mol\%) showed relatively faster blood clearance of DXR than standard liposomal DXR (Fig 1). This finding may reflect leakage of DXR from the liposome carrier due to high membrane permeability and consequent distribution to other tissues, and/or premature uptake of the DOPE-rich liposomes by the MPS. The DOPE-based liposomes showed no in vitro DXR leakage in $20 \%$ serum. However, the stability in vitro may not always reflect liposomal drug retention in vivo, due to e.g. differences in the composition of bovine serum and mice blood. Discrepancy between in vitro and in vivo leakage results has previously been observed (Charrois and Allen., 2004; Mayer et al., 1990).

Previous studies have demonstrated faster blood clearance kinetics of liposomal drugs composed of fluid lipids as a consequence of drug leakage (Charrois et al., 2004, Gabizon et al., 1988; Gabizon et al., 1993., Ishida et al., 2006). The permeability of liposome bilayers is to a great extent modulated by the $T_{c}$ of the phospholipids and the presence of cholesterol (Gabizon et al., 2003). DOPE has a $\mathrm{T}_{\mathrm{c}}$ of $-16^{\circ} \mathrm{C}$ (Cullis et al., 1978), resulting in highly fluid membranes at physiological temperature that are more permeable to molecules and ions. A partial loss of transmembrane ion-gradient might account for an elevated percentage of nonionized DXR in the liposome interior and subsequently DXR leakage.

Furthermore, a correlation between liposome permeability, lipid phase transition and cholesterol content has been established previously (Papahadjopoulos, 1996; Demel et al., 1976). In general, cholesterol is known to improve the physical stability of PC-based liposomes by tightening the lipid membrane, reducing its permeability and consequently leakage of entrapped drug (Demel et al., 1976). It was therefore interesting to note that no prolonged blood circulation time was obtained by increasing the cholesterol level from 20 to $40 \mathrm{~mol} \%$ in DOPE-rich liposomes. The lack of any cholesterol stabilizing effect might be related to membrane packing constrains such as inhomogeneous distribution of cholesterol within the lipid membranes and/or poor cholesterol-phospholipid interactions, leading to high membrane permeability (Demel et al., 1976).

When the DOPE level in liposomes was reduced to $32 \mathrm{~mol} \%$ or below, the amount of DSPC in the membrane was consequently increased. The significantly prolonged blood circulation time of DXR for these formulations was probably both a result of reduced levels of DOPE and increased levels of DSPC. Previous studies have shown that saturated phosphatidylcholine provides order and rigidity to fluid-like lipid membranes and prolongs the blood residence time of liposomal drugs due to reduced leakage from the carrier (Demel et al., 1976; Charrois et al., 2004; Gabizon et al., 1988; Ishida et al., 2006; Senior et al., 1982). 
The biodistribution studies indicates that the faster blood clearance for the DOPE-rich liposomal DXR was not the result of enhanced uptake by the MPS but due to increased leakage of DXR from the liposome carrier. This could be assumed as no increased uptake of DXR in liver and spleen was observed for the DOPE-rich liposomes, which displayed the lowest blood concentrations. On the contrary, slightly higher levels of DXR in liver and predominantly in kidneys were observed for the long circulating intermediatelevel DOPEcontaining liposomes. Considering slower release of DXR from the intermediatelevel DOPEcontaining liposomes in blood circulation, increased delivery of the liposome-encapsulated DXR to tissues would be expected. The significantly higher DXR concentrations in the kidneys at the latest time points after injection might reflect a sustained release of DXR in blood circulation. It should not be excluded, though, that the different blood clearance of liposomes comprising intermediate and high levels of DOPE also could be explained by different extent of uptake or accumulation in other organs or tissues not investigated here, such as the skin or paws.

There is a well-established correlation between long blood circulation time and increased liposome accumulation in tumours (Gill et al., 1995). Hence, the prolonged blood circulation time for the 25 and $32 \mathrm{~mol} \%$ DOPE-containing liposomes should provide a good basis for efficient tumour accumulation. To enable full availability of DXR to tumour cells, the DOPE-containing liposomes should be sufficiently destabilized by US at the tumour site. Interestingly, the in vitro sonosensitivity of liposomes was largely maintained down to a level of $25 \mathrm{~mol} \%$ DOPE. This particular formulation, comprising DOPE:DSPC:DSPEPEG:cholesterol 25:27:8:40 mol\%, showed approximately 70\% release of DXR after $6 \mathrm{~min}$ US exposure in $20 \%$ serum. The results suggest that DOPE is a very potent sonosensitivity modulator, as only small amounts of the lipid were required to achieve efficient US mediated drug release. The DOPE-liposomes represented a several fold improvement in sonosensitivity compared to DSPE-based liposomal DXR and standard liposomal DXR, respectively (Hagtvet et al., 2010; Evjen et al., 2011).

The US regime used in the current study ( $4 \min 40 \mathrm{kHz}$ US) is recently reported to induce a significant tumour regression in mice receiving DSPE-based liposomes, without inducing any acute toxicity (Hagtvet et al., 2010). However, for further preclinical and clinical studies of DOPE-containing liposomes a more sophisticated US device enabling increased focusing ability to tumours may be preferred. This will be accomplished by using high frequency focused US probes. However, previous studies have shown that sonosensitivity of the liposomes are comparable using $40 \mathrm{kHz}$ and $1.13 \mathrm{MHz}$ US (Evjen et al., 2011b). 
In conclusion, novel sonosensitive and long-circulating DXR-liposomes containing DOPE were developed by optimising the membrane composition. Low or moderate amounts of DOPE were required to obtain a sufficiently long blood circulation time after i.v. injection in mice. This reduction in DOPE level did not adversely affect sonosensitivity. Further studies are required to establish whether the novel formulations in combination with US improve DXR delivery to tumour cells enhancing the therapeutic index.

\section{ACKNOWLEDGEMENTS}

The project is supported by an industrial $\mathrm{PhD}$ grant from the Norwegian Research Council. The authors thank Deneke Fetene (Dept of Radiation Biology, Institute for Cancer Research, Oslo University Hospital), Sibylla Røgnvaldsson and Robert A. Fowler (Epitarget AS, Oslo), for technical assistance.

\section{REFERENCES}

Charrois, G.J.R., Allen, T.M., 2004. Drug release rate influences the pharmacokinetics, biodistribution, therapeutic activity, and toxicity of PEGylated liposomal doxorubicin formulations in murine breast cancer. Biochim Biophysic Acta. 1663,167-177.

Colbern, G., Vaage, J., Donovan, D., Uster, P., Working, P., 2000. Tumor uptake and therapeutic effects of drugs encapsulated in long-circulating PEGylated stealth liposomes. Journal of liposome research. 10, 81-92.

Cullis, P.R., Akshay, lagna P.R., de kruijff, B., 1978., The polymorphic phase behaviour of phosphatidylethanolamines of natural and synthetic origin. A 31P NMR study. Biochim Biophys Acta. 513, 31-42.

Damen, J., Regts, J., Scherphof, G., 1981. Transfer and exchange of phospholipid between small unilamellar liposomes and rat plasma high density lipoproteins. Dependence on cholesterol content and phospholipid composition. Biochim Biophys Acta. 665, 538-545.

Demel, R.A., De kruyff, B., 1976. The function of sterols in membranes. Biochim Biophys Acta. 457, 109-132.

de Smet, M., Heijman, E., Langereis, S., Hijnen, N., Grull, H., 2010. Mangnetic resonance imaging of high intensity focused ultrasound mediated drug delivery from temperaturesensitive liposomes: An in vivo proof-of-concept study. Journal of Controlled Release. In press.

Drummond, D.C., Meyer, O., Hong, K., Kirpotin, D.B., Papahadjopoulos, D., 1999. Optimizing liposomes for delivery of chemotherapeutic agents to solid tumours. Pharmacological Reviews. 51, 691-743.

Evjen, T.J., Nilssen, E.A., Røgnvaldsson, S., Brandl, M., Fossheim, S.L., 2010. Distearoylphosphatidylethanolamine-based liposomes for ultrasound-mediated drug delivery. European Journal of Pharmaceutics and Biopharmaceutics. 75, 327-333. 
Evjen, T.J., Nilssen, E.A., Barnert, S., Schubert, R., Brandl, M., Fossheim, S.L., 2011 a. Ultrasound-mediated destabilization and drug release from liposomes comprising dioleoylphosphatidylethanolamine. European Journal of Pharmaceutical Sciences. 42, 380386.

Evjen, T.J., Nilssen, E.A., Fowler, R.A, Røgnvaldsson, S., Brandl, M., Fossheim, S.L., 2011 b. Lipid membrane composition influences drug release from dioleoylphosphatidylethanolamine-based liposomes on exposure to ultrasound. International Journal of Pharmaceutics. 406, 114-116.

Ferrara, K., 2008. Driving delivery vehicles with ultrasound. Adv Drug Deliv Rev. 60, 10971102.

Frenkel, V., 2008. Ultrasound mediated delivery of drugs and genes to solid tumors. Adv Drug Deliv Rev. 60, 1193-1208.

Gabizon, A., Papahadjopoulos, D., 1988. Liposome formulations with prolonged circulation time in blood and enhanced uptake by tumours. Proceedings of the National Academy of Sciences of the United States of America. 85, 6949-6953.

Gabizon, A., Shiota, R., Papahadjopoulos, D., 1989. Pharmacokinetics and tissue distribution of doxorubicin encapsulated in stable liposomes with long circulation times. J Natl Cancer Inst. $81,1484-1488$.

Gabizon, A., Barenholz, Y., Bialer, M., 1993. Prolongation of the circulation time of doxorubicin encapsulated in liposomes containing a polyethylene glycol-derivatized phospholipid: pharmakokinetic studies in rodents and dogs. Pharmaceutical Research. 10, 703-708.

Gabizon, A., Scmeeda, H., Barenholz, Y., 2003. Pharmacokinetics of PEGylated liposomal doxorubicin. Review of animal and human studies. Clin Pharmacokinet. 42, 419-436.

Gill, P.S., Espina, B.M., Muggia, F., Cabriales, S., Tulpule, A., Esplin, J.A., Liebman, H.A., Forssen, E., Ross, M.E., Levine, A.M., 1995. Phase I/II clinical and pharmacokinetic evaluation of liposomal daunorubicin. J Clin Oncol. 13, 996-1003.

Gregoriadis, G., 1988. Fate of injected liposomes: observations on entrapped solute retention, vesicle clearance and tissue distribution in vivo. Liposomes as drug carriers, ed., Chichester: John Wiley \& sons Ltd. p 3-18.

Hagtvet, E., Evjen, T.J., Olsen, D.R., Fossheim, S., Nilssen, E.A., 2010. Ultrasound enhanched antitumour activity of liposomal doxorubicin in mice. Journal of drug targeting. In press.

Haran, G., Cohen, R., Bar, L.K., Barenholz, Y., 1993. Transmembrane ammonium sulfate gradients in liposomes produce efficient and stable entrapment of amphipathic weak bases. Biochim Biophys Acta. 1151, 201-215.

Horowitz, A.T., Barenholz, Y., Gabizon, A.A., 1992. In vitro cytotoxicity of liposomeencapsulated doxorubicin: dependence on liposome composition and drug release. Biochim Biophys Acta. 1109, 203-209.

Huang, S.-L., MacDonald, R.C., 2004. Acoustically active liposomes for drug encapsulation and ultrasound-triggered release. Biochim Biophys Acta, 1665, 134-141. 
Huang, S.-L., McPherson, D.D., MacDonald, R.C., 2008. A method to co-encapsulate gas and drugs in liposomes for ultrasound-controlled drug delivery. Ultrasound in medicine and biology, 34, 1272-1280.

Ishida, T., Okada, Y., Kobayashi, T., Kiwada, H., 2006. Development of pH-sensitive liposomes that efficiently retain encapsulated doxorubicin (DXR) in blood. International Journal of Pharmaceutics. 309, 94-100.

Lin, H.,-Y., Thomas, J.L., 2004. Factors affecting responsitivity of unilamellar liposomes to $20 \mathrm{kHz}$ ultrasound. Langmuir. 20, 6100-6106.

Maeda, H., Wu, J., Sawa, T., Matsumura, Y., Hori, K., 2000. Tumor vascular permeability and the EPR effect in macromolecular therapeutics: a review. J Controlled release. 65, 271284.

Mayer, L.D., Bally, M.D., Loughrey, H., Masin, D., Cullis, P.R., 1990. Liposomal vincristine preparations which exhibit decreased drug toxicity and increased activity against murine $\mathrm{L}$ 1210 and P388 tumors. Cancer research. 50, 575-579.

Myhr, G., Moan, J., 2006. Synergistic and tumour selective effects of chemotherapy and ultrasound treatment. Cancer letters. 232, 206-213.

Papahadjopoulos, D., 1996. Fate of liposomes in vivo: A brief introductory review. Journal of liposome research. 1, 3-17.

Pitt, W.G., Husseini, G.A., Staples, B.J., 2004. Ultrasonic Drug Delivery- A General Review. Expert Opin Drug Deliv. 1, 37-56.

Pong, M., Umchid, S., Guarino, A.J., Lewin, P.A., Litniewski, J., Nowichi, A., Wrenn, S.P., 2006. In vitro ultrasound- mediated leakage from phospholipid vesicles. Ultrasonics. 45, 133 145.

Schroeder, A., Kost, J., Barenholz, Y., 2009. Ultrasound, liposomes, and drug delivery: principles for using ultrasound to control the release of drugs from liposomes. Chemistry and Physics of Lipids. 162, 1-16.

Senior, J., Gregoriadis, G., 1982. Stability of small unilamellar liposomes in serum and clearance from the circulation: The effect of the phospholipid and cholesterol components. Life sciences. 30, 2123-2136.

Unger, E.C., McCreery, T.P., Sweitzer, R.H., Caldwell, V.E., 1998. Acoustically active lipospheres containing paclitaxel: A new therapeutic ultrasound contrast agent. Investigative radiology. 33, 887-892. 